\title{
ANISOTROPIC MESOSCOPIC TRAFFIC SIMULATION APPROACH TO SUPPORT LARGE- SCALE TRAFFIC AND LOGISTIC MODELING AND ANALYSIS
}

\author{
Ye Tian \\ Yi-Chang Chiu \\ Department of Civil Engineering and Engineering Mechanics \\ The University of Arizona \\ $1209 \mathrm{E} 2^{\text {nd }}$ St. \\ Tucson, AZ, 85721, USA
}

\begin{abstract}
Large-scale traffic and transportation logistics analysis requires a realistic depiction of network traffic condition in a dynamic manner. In the past decades, vehicular traffic simulation approaches have been increasingly developed and applied to describe time-varying traffic dynamics. Most of the existing approaches are so-called microscopic simulation in which complex driving behaviors such as car following and lane-changing are explicitly modeling in second or sub-second time resolution. These approaches are generally challenging to calibrate and validate and they demand a vast amount of computing resources. This paper discusses a new Anisotropic Mesoscopic Simulation (AMS) approach that carefully omits micro-scale details but nicely preserves critical traffic dynamics characteristics. The AMS model allows computational speed-ups in the order of magnitudes compared to the microscopic models, making it well-suited for large-scale applications. The underlying simulation rules and macroscopic dynamical characteristics are presented and discussed in this paper.
\end{abstract}

\section{INTRODUCTION}

Large-scale traffic and transportation logistics analysis requires a realistic depiction of network traversal time in a dynamic manner. In the past decades, vehicular traffic simulation approaches have been increasingly developed and applied to describe time-varying traffic dynamics. Most of the existing approaches are so-called microscopic simulation in which complex driving behaviors such as car following and lane-changing are explicitly modeling in second or sub-second time resolution. The research developments related to microscopic traffic simulation models can be generally classified into theoretical development (Ferreira et al. 2009; Kerner et al. 2006; Burghout et al. 2005), model calibration and validation (Simith et al. 2006; Park et al. 2006; Ozbay et al. 2006), and integrated applications (Yin et al. 2007; Shelton 2008; Zhang et al. 2010).

Another class of traffic simulation model is mesoscopic, which is aimed at balancing computational burden and realism of macroscopic simulation properties by selectively omitting certain car-following behavior and adopting a coarser simulation temporal resolution. In the field of quantum physics, the term mesoscopic can be generally defined in the field of quantum physics as: "pertaining to a size regime, intermediate between the microscopic and the macroscopic, that is characteristic of a region where a large number of particles can interact in a quantum-mechanically correlated fashion." (McGraw-Hill et al. 2002) For traffic modeling, mesoscopic can be understood as time scale at which one can reasonably discuss the properties of a phenomenon without having to discuss the behavior of individual vehicles, and concepts of averages are useful. Based on this principle, a wide and diversified range of models can be considered mesoscopic models.

In the literature earlier models that generally considered mesoscopic include the headway distribution model that assumes probability distribution functions to capture vehicle traffic dynamics (Buckley 1968; 
Branston 1976); the cluster model (Botma 1978); and the gas-kinetic continuum model (Nelson et al. 1998). Another model class is the simulation-oriented models that are aimed at describing vehicle trajectories following link, segment, or cell structure of the network arcs at every simulation clock tick. Falling within this category are several other types of models with distinct modeling features. A common model type is the link-based queue-server models such as the Queue model introduced by (Gawron 1998) and the late on proposed by (Cetin et al. 2002).

In another type of model in the same category, vehicles are moved according to an average speed calculated from the average link density in conjunction with a macroscopic speed-density $(v-k)$ relationship at every clock tick. A queue-server at the downstream node discharges vehicles according to the constraints imposed at these nodes, accounting for delays caused by traffic signals, downstream capacity limits, and interaction with additional traffic. A typical model requires a non-zero minimal speed to prevent a complete stop once the link density reaches the jam density. A vehicle's change of speed when moving from one upstream link to the downstream link is not explicitly regulated. A typical model is DYNASMART (Jayakrishnan et al. 1994).

Such models often become an integral part of simulation-based dynamic traffic assignment (SBDTA) for traffic modeling at scale larger than that for microscopic models. Its macroscopic relation, which aims to maintain reasonable traffic flow dynamic, is utilized to evaluate the time-dependent link travel time and intersection turning penalties - solving the intermediate dynamic traffic assignment solutions during the solution process (Ben-Akiva et al. 2001; Taylor 2003; Mahmassani et al. 1993; Mahmassani 2001; BenAkiva et al. 2003; Burghout et al. 2005). The main drawback of this class of model, as well discussed in several studies is the lack of anisotropic properties (Dell'Orco 2006; Zhang 2001; Daganzo 1995). The anisotropic property means that vehicles react to traffic condition mostly in the front so the stimulus for vehicle reactions is directional. The above mentioned models may exhibit anomalous behavior under certain common conditions (e.g. increased inflow from upstream may slow down vehicles downstream.)

The Anisotropic Mesoscopic Simulation (AMS) model developed by Chiu et al. (Chiu et al. 2010) for the uninterrupted flow facility such as freeway systems was demonstrated to exhibit desirable traffic macroscopic dynamic properties in real-world applications (Shelton et al. 2009; Alexiadis 2008; Shelton 2007; Kuhn et al. 2008; Zheng et al. 2010; Noh et al. 2009; Kary et al. 2009). The underlying idea of this model is that each vehicle's prevailing speed at current time is determined by a response function which takes an average density in the front of the following vehicle as the function input. Several desirable traffic flow properties arise from this simple formulation. This paper reviews the general AMS model specification and applications as well as recent development of the lane-based enhancement of AMS mode. Current field applications of AMS based model are also discussed.

\section{ANISOTROPIC MESOSCOPIC SIMULATION (AMS) MODEL}

Two intuitive concepts and traffic characteristics form the foundation of AMS: (1) at any time, a vehicle's prevailing speed is influenced only by the vehicles in front of it, including those that are in the same or adjacent lanes; (2) the influence of traffic downstream upon a vehicle decreases with increased distance. These two characteristics define the "anisotropic" property of the traffic flow and provide the guiding principle for AMS model design. Based on such concepts, we define that for any vehicle $i$, only those leading vehicles (in the same lane or in the adjacent lanes) present in vehicle $i$ 's immediate downstream and within a certain distance are considered to influence vehicle $i$ 's speed response. This is a similar concept to a stimulus-response type of car-following model, with the distinction that in AMS, the stimulus of a vehicle's speed response is represented in a macroscopic manner instead of using intervehicle distance or speed as in microscopic models.

For modeling purposes, the Speed Influencing Region for vehicle $i\left(S I R_{i}\right)$ is defined as vehicle $i$ 's immediate downstream roadway section in which the stimulus is significant enough to influence vehicle $i$ 's speed response. This concept is further depicted in Figure 1, in which a multi-lane homogeneous roadway segment is considered. The Speed Influencing Region (SIR) for vehicle $i$ is defined as the area (including the lane in which vehicles reside and all the adjacent lanes) in front of vehicle $i$, where the 
traffic condition (represented by the density) affects vehicle $i$ 's speed response. At each simulation clock tick, vehicle $i$ 's speed is influenced by the density in SIR. The upstream traffic and downstream traffic outside the $S I R$ does not influence vehicle $i$. The $S I R_{i}$ length can be assumed to be either equal for all vehicles or variable according to different flow conditions. The $S I R_{i}$ length is assumed to be an average value $l$ across all vehicles in this paper. The traffic density in $S I R_{i}$, denoted as $k_{i}$, is calculated as the number of vehicles present in $S I R_{i}$ divided by the total lane-miles of the $S I R_{i}$. As such, the unit of $k_{i}$ becomes the number of vehicles per mile per lane.

At the beginning of a simulation interval $t$, for each vehicle $i$, the prevailing speed of vehicle $i$ during the simulation interval $t$ is determined by Equation (1), where $\wp: k \rightarrow v$ is a non-increasing speeddensity relationship function with two boundary conditions: $\wp(0)=v_{f}$ and $\wp\left(k_{\text {queue }}\right)=0$. The queue density $k_{\text {queue }}$ is defined as the "bumper-to bumper" density observed in a long, standing-still queue, which is generally greater than the jam density reported in the literature.

The algorithmic steps of an AMS model during simulation are as follows: at each clock tick $t$ (the beginning of a simulation interval), each vehicle's speed $v_{i}^{t}$ is evaluated based on its $S I R$ density, which is obtained from the previous clock tick $k_{i}^{t-1}$ through the $v-k$ relationship function $\wp\left(k_{i}^{t-1}\right)$ shown in Equation (4). The SIR density is calculated based on Equations (2) or (3), depending on whether or not the $S I R$ spans over the freeway segment with a different capacity. If the SIR spans a homogeneous highway section, Equation (2) applies; otherwise, Equation (3) is used. Vehicle $i$ 's traveling distance at the end of the current simulation interval is obtained by taking the prevailing speed $v_{i}^{t}$ times the duration of the simulation interval $\Delta$.

$$
\begin{aligned}
& v_{i}^{t}=\wp\left(k_{i}^{t-1}\right) \\
& k_{i}^{t-1}=\min \left[k_{\text {queue }}, \frac{N_{i}^{t-1}}{n l}\right] \\
& k_{i}^{t-1}=\min \left[k_{\text {queue }}, \frac{N_{i}^{t-1}}{m x_{i}^{t-1}+n\left(l-x_{i}^{t-1}\right)}\right] \\
& v^{c a l}=\left\{\begin{array}{cl}
v_{f} & k \leq k_{b} \\
v_{f}\left[1-\left(\frac{k-k_{b}}{k_{j a m}-k_{b}}\right)^{\beta}\right]^{\alpha} & k_{b} \leq k \leq k_{j a m}
\end{array}\right.
\end{aligned}
$$




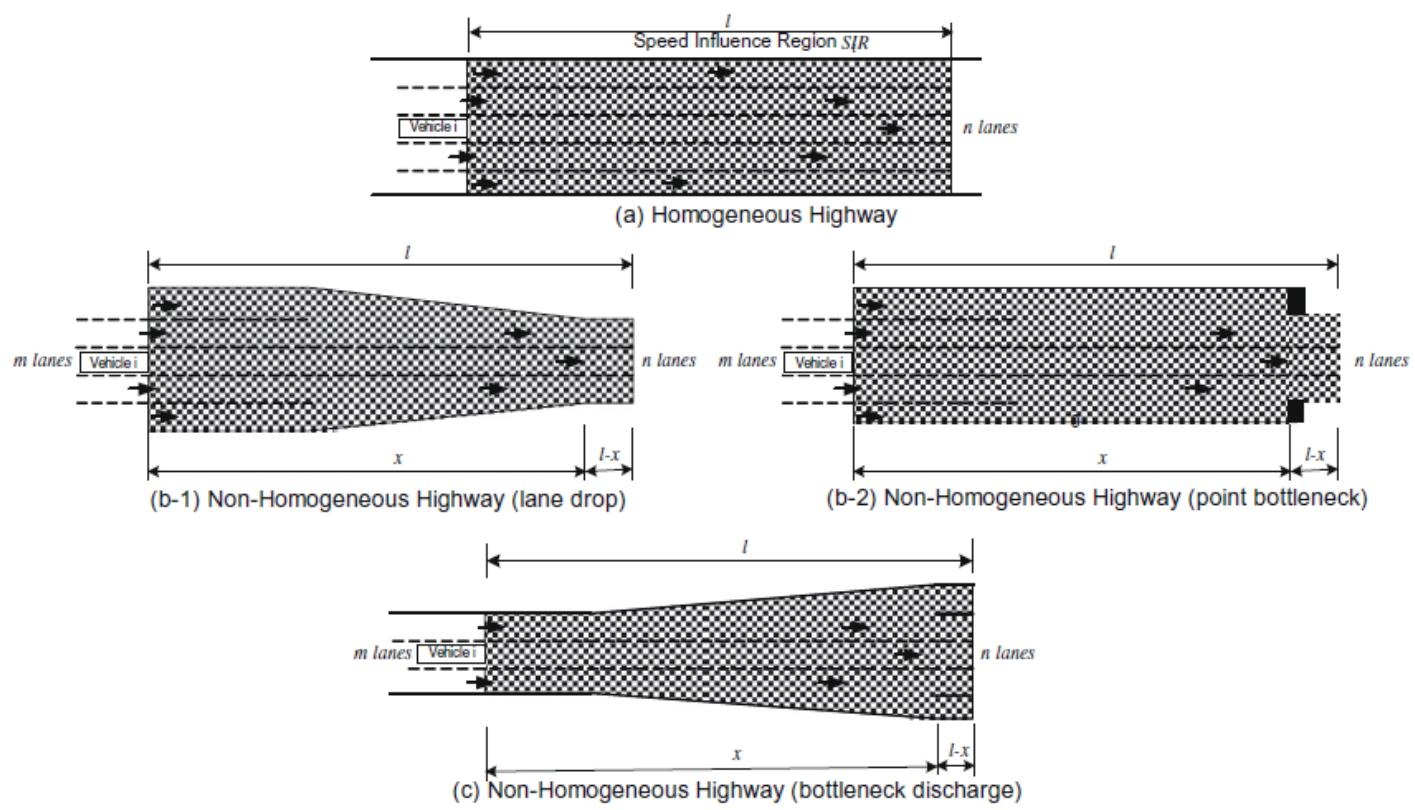

Figure 1: AMS Concept (Chiu et al. 2010)

The basic AMS model proposed by Chiu et al. (2010) is not a lane-specific model in which no delineation of traffic lanes is specified. The numerical analysis with lane drops and blockage provided in (Chiu et al. 2010) conforms to desirable microscopic traffic properties without explicitly modeling lane changing. At times, lane-specific treatment may become desirable under certain geometric (e.g. freeway weaving section, off-ramp) or operating conditions (e.g. a stopped bus blocking traffic in the back or traffic spills back from a freeway off-ramp). From a hybrid or adaptive simulation standpoint, allowing additional simulation logics to be applied in different traffic condition to form an Adaptive Simulation (AS) strategy appears to be advantageous in a simulation environment with heterogamous environment and requirements.

\subsection{Lane-Changing Model for AMS}

Lane changing is a basic car-following behavior when the following vehicle catches up with a slower moving lead vehicle and the adjacent lane permits acceptable gap. By switching to the adjacent lane the following vehicle is able to maintain the driver's desirable speed. All microscopic traffic simulation models include lane-changing rules as the essential driving logics (Bloomberg et al. 2000; Cheu et al. 2004; Nagel et al. 1997; Yang et al. 1996). In a typical cellular automata model, the lane changing rule is based on the relationship between several different "gaps" (Nagel 1996). Gap means the time distance measure between two consecutive vehicles passing the same reference point. Cheu et al. (2009) implemented the lane changing behavior in Cell Transmission Model (CTM) when trying to simulate the Port of Entry (POE) vehicles. The proposed model can plot out the vehicle trajectory and lane density as well. Laval et al. (Laval et al. 2006) proposed a lane-changing model that uses four easy-to-measure parameters and the model was test on two different phenomena which are: the drop in discharge rate of freeway bottlenecks when congestion begins and the relation between the speed of a moving bottleneck and its capacity.

The lane changing model for AMS model studied herein considers lane changing behavior as a quite complex procedure. As a result, several internal and external factors should be taken into account:

1. The triggers for the following vehicle driver to consider changing lane in AMS include one or several following conditions:

a. The speed of the lead vehicle is slower than the desired speed of the following vehicle, 
b. There is a sufficient gap between the nearest lead vehicle in the neighboring lanes,

c. The vehicle in the neighboring lane is moving faster than the lead vehicle, and

d. The lane the following vehicle is in is going to merge into the neighbor lane and the vehicle has to change lane.

2. The opportunity to change lane conforms to the safety consideration, which is the nearest vehicle upstream upon the lane changing considered vehicle in the neighboring lane is sufficiently far away so that the vehicle can change lane freely without concerning possible crash.

The original AMS model is enhanced with the following lane changing procedures:

1. Implement the lane changing model in AMS,

2. Update the speed based on the SIR density. To this end the SIR density is weighted by the current and neighboring lane density. The weight for the current lane should be higher than the neighboring lane, representing varying degree of influence between the two lanes. An example is the weight of the current lane is $70 \%$ and $30 \%$ for the neighboring lane as shown in Equation (5), and

$$
v_{i}^{t}=\wp\left(k_{i}^{t-1}\right), k_{i}^{t-1}=\min \left[k_{\text {queue }}, \frac{N_{i}^{t-1}+I_{L} N_{i(L)}^{t-1}+I_{R} N_{i(R)}^{t-1}}{\left(1+I_{L}+I_{R}\right) l}\right]
$$

3. Update the position of the following vehicle. If the updated position of the following vehicle $i$ precedes the lead vehicle, then remove the speed data of vehicle $i$ calculated in procedure 2), set the speed of vehicle $i$ to be the forward gap in current lane, then update the position using the new speed data.

The lane changing action and forward movement updates are performed in separate algorithmic iterations. The first iteration updates the intended speed and position of all vehicles on the same link. The second iteration updates the position of all vehicles. Each vehicle is making its driving decision based on the configuration at the beginning of the update.

The AMS lane changing algorithm is expressed as follows, assuming that the simulation step length is $s$ sec., the unit of speed is feet per second and the unit of all the length is feet:

1. Calculate three gaps:

$\operatorname{gap}(i)$ Gap Forward in current lane

gapo(i) Gap Forward in the neighboring lane

$\operatorname{gapb}(i)$ Gap Backward in the neighboring lane

2. Calculate weight ${ }^{1}$ to weight ${ }^{3}$ based on the gap values

Weight $^{1}=\operatorname{speed}(i)-\operatorname{gap}(i)$

Weight $^{2}=\operatorname{gapo}(i)-\operatorname{speed}(i)$

Weight $^{3}=\operatorname{gapb}(i)-$ MaxSpeed

Where MaxSpeed is the attainable max vehicle speed, which is equivalent to the free-flow speed. The MaxSpeed is assumed to be identical for all vehicles on the same link. This can be relaxed if a higher degree of heterogeneity is needed.

3. If weight ${ }^{1}$, weight ${ }^{2}$ and weight ${ }^{3}$ are all larger than zero, then mark the vehicle as "lane-changing", change the value of gap $(i)$ and gapo $(i)$.

For the planned lane changing in order to traverse the point bottleneck, the lane changing rules would be different in two segments. Before the location of the "Lane Blocked" warning sign (Segment I in Figure 2), the lane changing rule is not activated. Assume that all the drivers notice the traffic warning (this assumption can be relaxed), after seeing the warning sign (Segment II in Figure 2), the lane change rule is activated and the following procedure (suppose there are two lanes and lane 1 is the open lane and lane 2 is blocked somewhere downstream):

1. Calculate three gaps, they are: $\operatorname{gap}(i), \operatorname{gapo}(i), \operatorname{gapb}(i)$ 
2. Calculate weight ${ }^{1}$ to weight ${ }^{4}$ based on the gap values

$$
\begin{aligned}
\text { Weight }^{1} & =\operatorname{speed}(i)-\operatorname{gap}(i) \\
\text { Weight }^{2} & =\operatorname{gapo}(i)-\operatorname{speed}(i) \\
\text { Weight }^{3} & =\operatorname{gapb}(i)-\operatorname{MaxSpeed}
\end{aligned}
$$

3. If the vehicle is in lane 1 , then:

If weight ${ }^{1}>a$, weight ${ }^{2}>b$, and weight ${ }^{3}$ is larger than 0 , then mark the vehicle as "lane changing"

If the vehicle is in lane 2, then:

If weight ${ }^{2}$ and weight ${ }^{3}$ are both larger than 0 , then mark the vehicle as "lane changing"

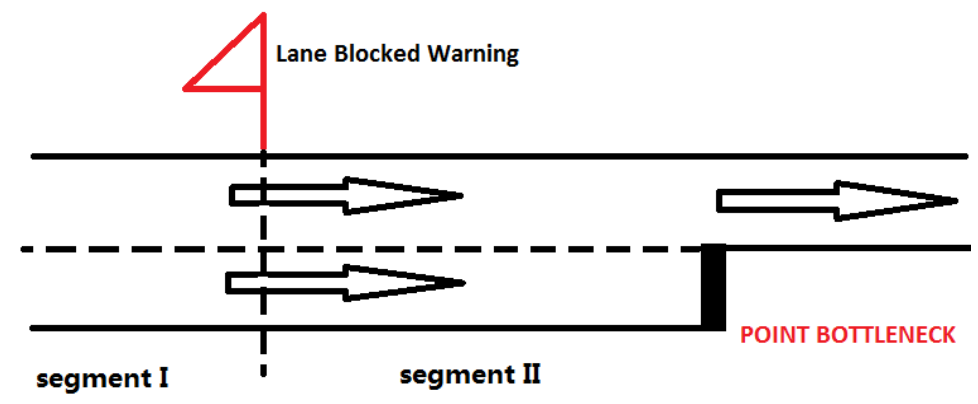

Figure 2 : Lane Blockage Scenario

Parameters $a$ and $b$ measure the lane 1 drivers' willing to speed up knowing the merge location is coming up. The smaller $a$ and $b$ are, the more likely that drivers in lane 1 want to change to lane 2 thought they knows lane 2 is going to be blocked somewhere downstream. In an extreme case, if $a$ and $b$ are both set to be zero, the drivers in lane 1 doesn't care about the point bottleneck ahead of them at all.

\section{NUMERICAL EXPERIMENTS}

Several different scenarios in a two-lane highway system are considered herein:

\subsection{Scenario 0 : Baseline Scenario}

Consider a 1-mile four-lane highway segment (two lanes in each direction) with the flow rate to be 1500 $\mathrm{veh} / \mathrm{ln} / \mathrm{hr}$, vehicle arrival follows a Poisson distribution with the average time headway $2.4 \mathrm{sec}$. The freeflow speed $v_{f}$ for each vehicle is assumed to be $89.91 \mathrm{feet} / \mathrm{sec}$ and all the other parameters for the modified Greenshield's speed-density function are set as following and the SIR length is set to be 225 feet according to Chiu et al. (2010).

$\begin{array}{cl}k_{b} & 31.361 \\ k_{\text {jam }} & 185.266 \\ \alpha & 1.94 \\ \beta & 0.5\end{array}$

Figure 3 displays the trajectories for 100 vehicles for this baseline scenario simulated by AMS lane changing. Because vehicles are traveling at the same free-flow speed, no lane changing occurs and all trajectories are in parallel. The total travel time for all the 100 vehicles to traverse this 1 mile segment is $5872 \mathrm{sec}$. 


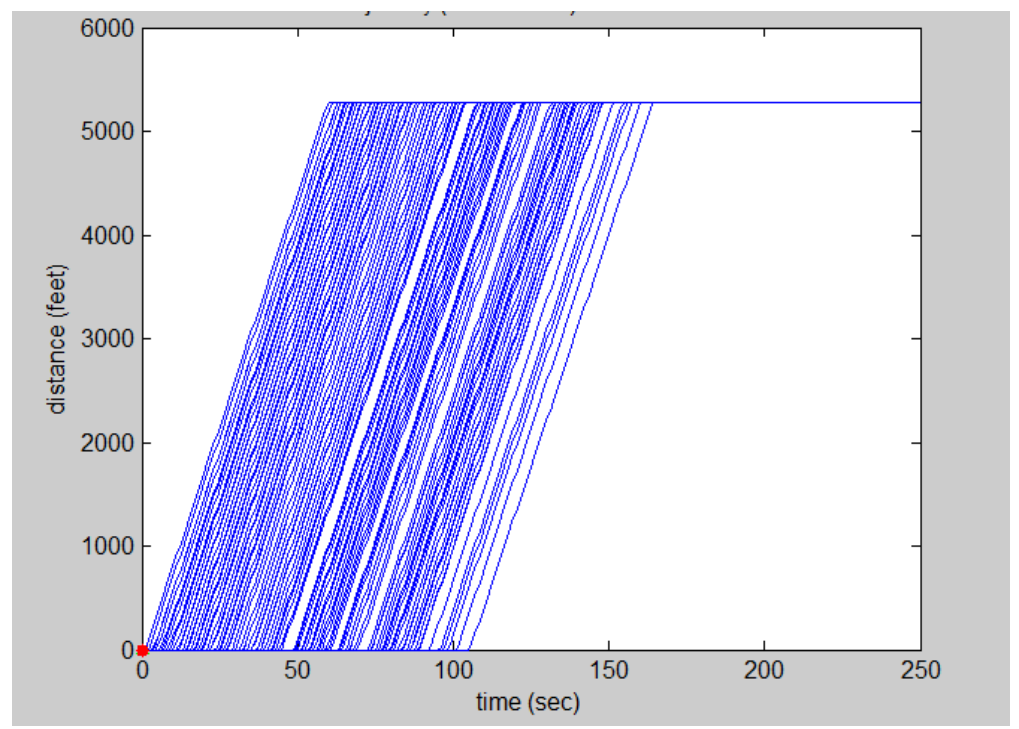

Figure 3: Baseline Scenario

\subsection{Scenario I : Stalled Vehicle}

Consider a 1-mile highway segment and 100 vehicles in total, one vehicle stalled in lane 2 for 50 seconds at the location of 4000 feet downstream. The flow remains $1500 \mathrm{veh} / \mathrm{ln} / \mathrm{hr}$.

Figure 4 shows the trajectory of vehicles in both lanes with the density weights for lanes 1 and 2 set to be 0.5 respectively. The green triangles represent vehicle moving from lane 1 to lane 2 (open lane to closed lane) and the red circles represent vehicles changing from lane 2 to 1 . From the graph one would notice that all the vehicles are in a stable status until approaching the incident location. All the vehicles in lane 2 (the stalled vehicle is in lane 2) would wait behind the stalled vehicle and the queue starts building up (because the event is no-noticed). Vehicles in lane 2 then start to look for an opportunity (the backward distance in the other lane is large enough) to change to lane 1 to overtake the stalled car and in the downstream of the incident location some vehicles in lane 1 would change back to lane 2 . The total travel time for all the vehicles is 7276 seconds, indicating a 1404 second delay compared to the baseline scenario. In Figure 4 we can also observe the queue building up and the dissipation process. The expected interfaces of the different conditions are quite clear.

Figure 5 shows the result of the case with different weights for different lanes when computing density using AMS model. In this case, the weight for the current lane in is $70 \%$ and $30 \%$ for the neighboring lane. Since the weight for the current lane is increased, when approaching the stalled vehicle, vehicles in lane 1 didn't experience a less sharp speed decrease compared to the previous case. Figure 6 shows the scenario when no lane changing behavior is allowed. All the vehicles in lane 2 behind the stalled vehicle have to wait in lane 2 till the stalled vehicle is moved away so much more delay is expected in this scenario. 
Tian and Chiu

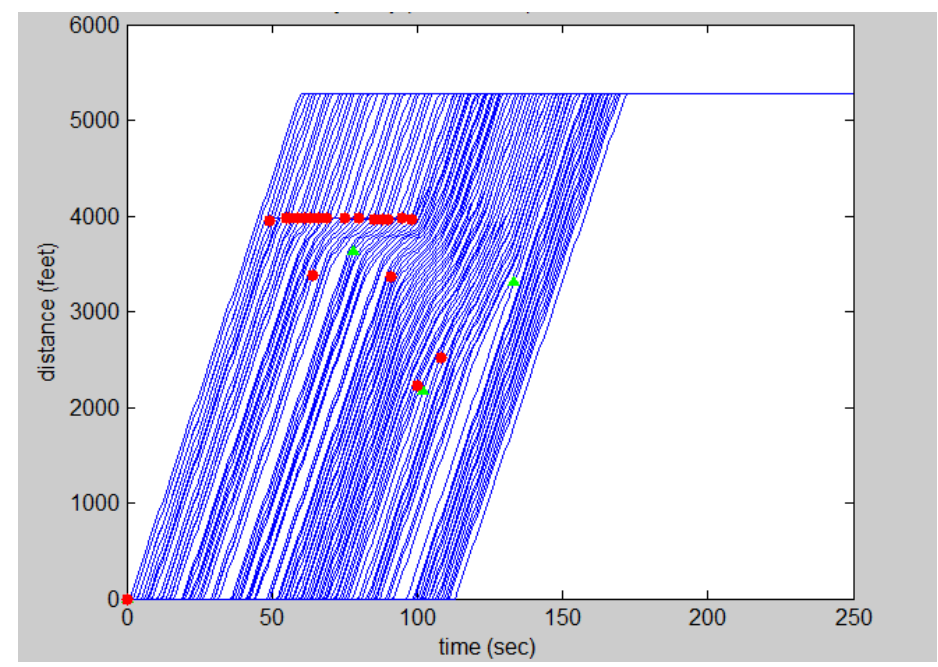

Figure 4: Equal Density Weight Scenario

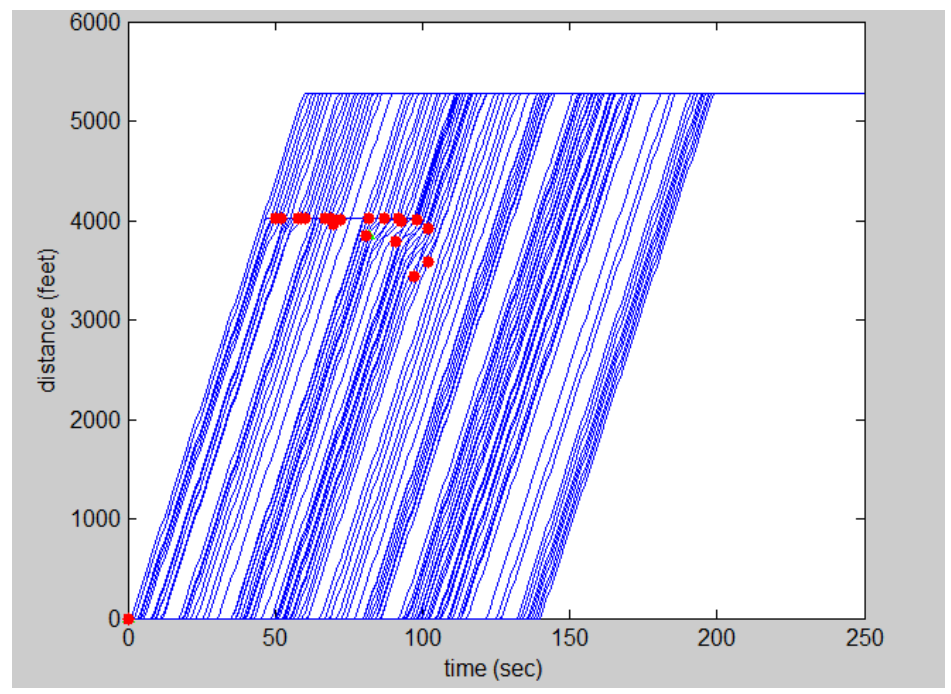

Figure 5: 70\%-30\% Density Weight Scenario

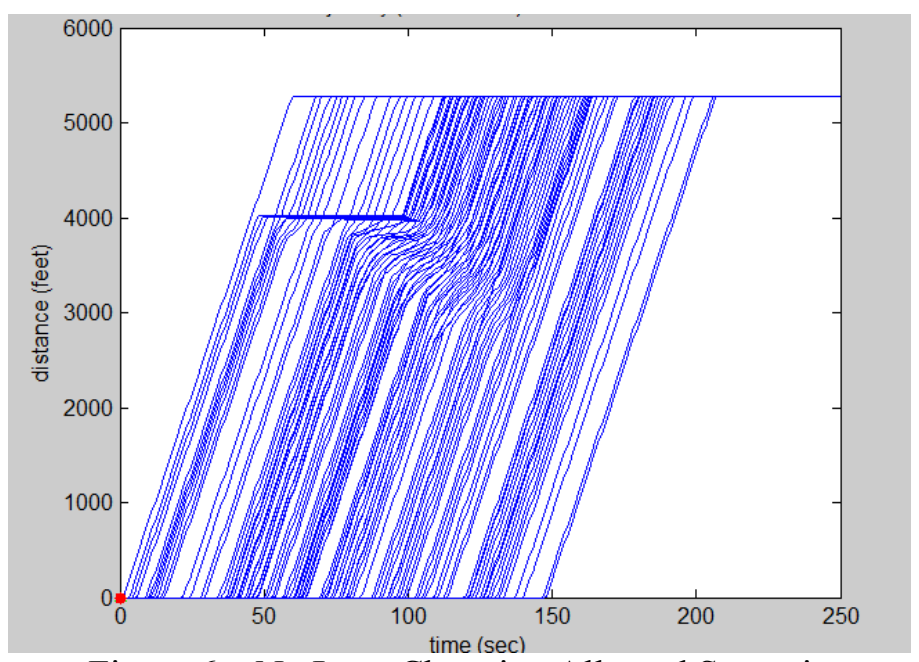

Figure 6: No Lane Changing Allowed Scenario 


\subsection{Scenario II: Slow Moving Vehicle}

In this scenario, a slow-moving vehicle is assumed to enter the freeway starting from the entrance 4000 feet downstream occupying one lane and exit the freeway after traveling 500 feet on the freeway.

Still consider a 1 mile segment and 100 vehicles in total, the speed of the slow moving vehicle is set to be $10 \mathrm{feet} / \mathrm{sec}$ and $25 \mathrm{feet} / \mathrm{sec}$ for comparison. The flow remains to be $1500 \mathrm{veh} / \mathrm{ln} / \mathrm{hr}$. The total delay for the $10 \mathrm{feet} / \mathrm{sec}$ scenario is $750 \mathrm{sec}$ and for the $25 \mathrm{feet} / \mathrm{sec}$ scenario, the delay is $68 \mathrm{sec}$ which is much smaller than the former scenario, indicating that a moving blockage with higher speed would cause less delay. Figure 9 and Figure 10 show the vehicle trajectories for the case without lane changing allowed. More delay can be observed in these two figures.

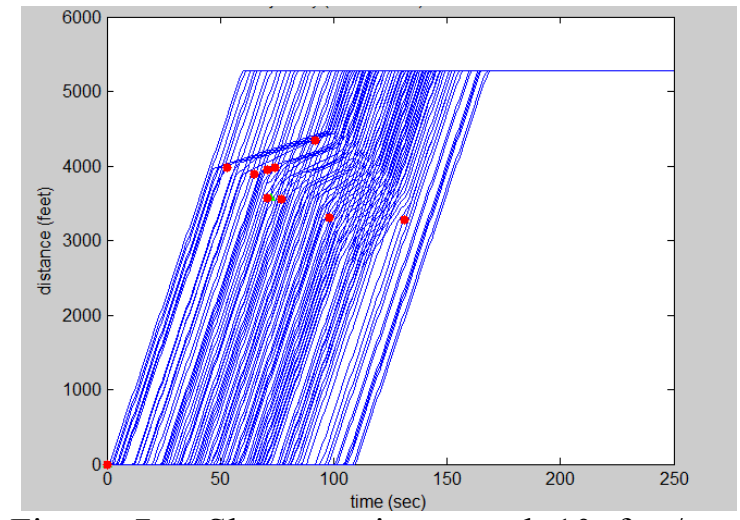

Figure 7: Slow moving speed 10 feet/sec; weights for the two lanes are $90 \%-10 \%$

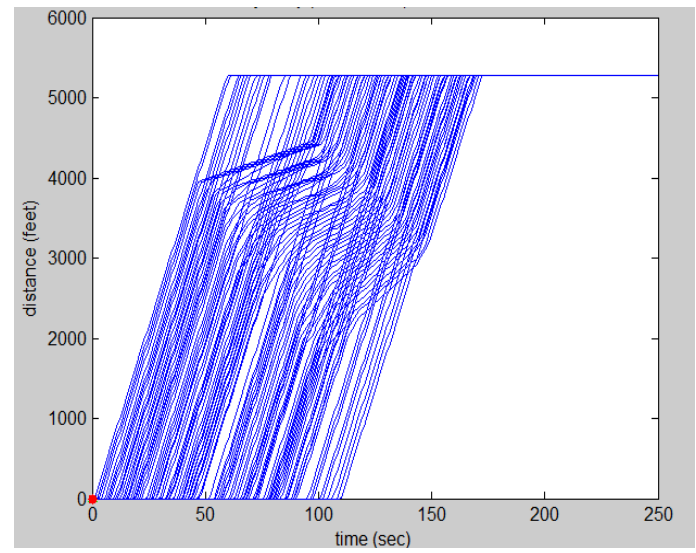

Figure 9: Slow moving speed 10 feet/sec; weights for the two lanes are $90 \%-10 \%$, no lane changing allowed

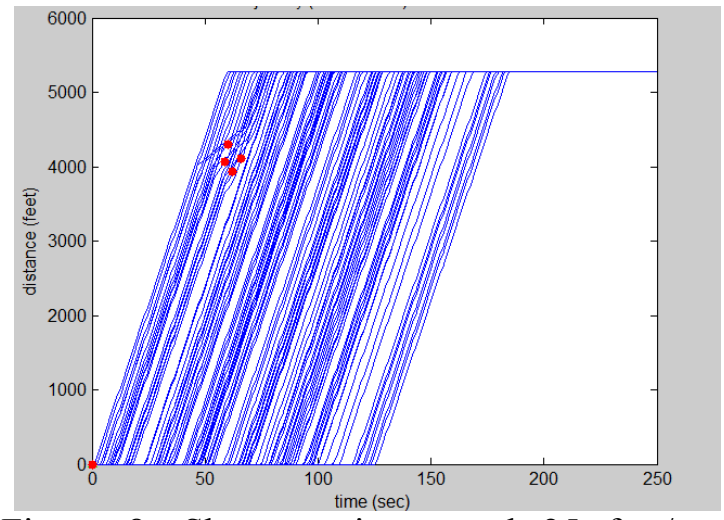

Figure 8: Slow moving speed 25 feet/sec; weights for the two lanes are $90 \%-10 \%$

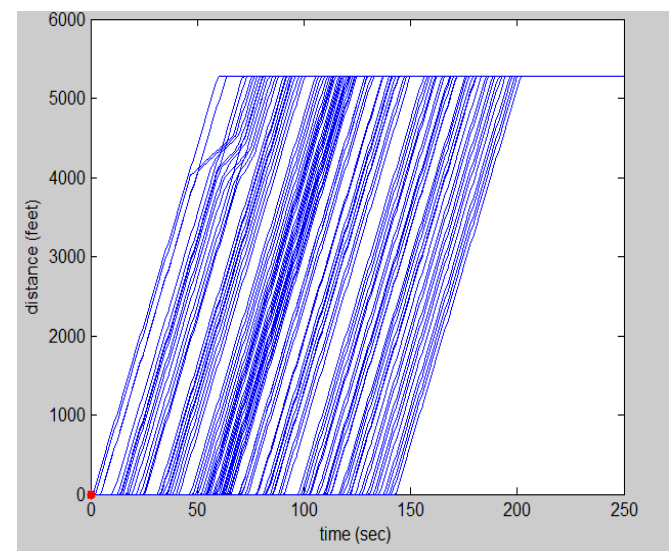

Figure 10: Slow moving speed 25 feet/sec; weights for the two lanes are $90 \%-10 \%$, no lane changing allowed.

\subsection{Scenario II: Highway Work Zone}

Suppose a highway work zone closes one of the two lanes, two different conditions are concerned and simulated: with and without a work-zone warning sign upstream of the bottleneck.

All the background settings remain unchanged except for a work zone occupying lane 2 starting at the 4000 feet downstream. At that location, two lanes taper and merge into one lane. The work zone is assumed to last till the downstream end of the studied segment. The warning sign is placed 1000 feet upstream of the work zone. 
The results show that the total delay for the scenario with warning sign is $1000 \mathrm{sec}$ which is $820 \mathrm{sec}$ less than the delay of without the warning sign. Less congestion can be observed in the warning sign scenario at the same time, which indicates that the warning sign ahead of the incident location helps alleviate the system congestion. Figure 13 shows the result with no lane changing allowed.

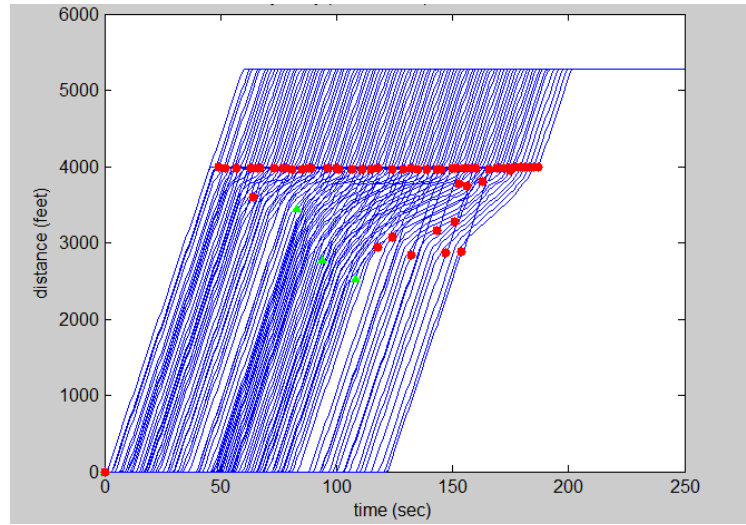

Figure 11: Work zone without warning sign scenario; density weight are $90 \%-10 \%$.

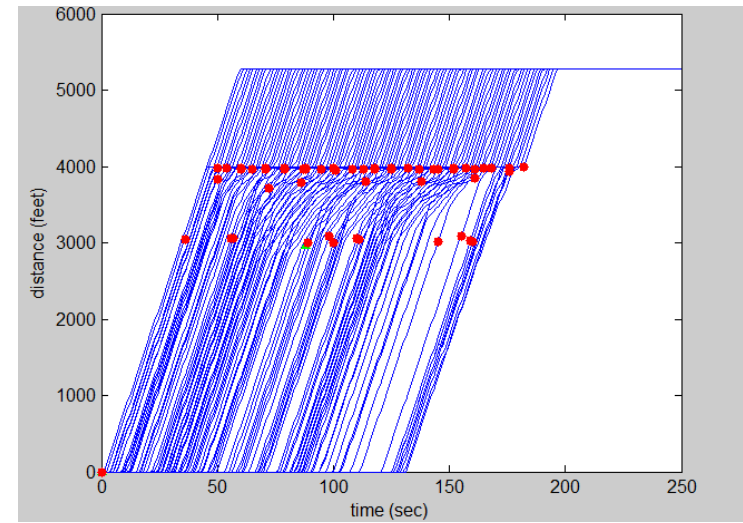

Figure 12: Work zone with warning sign; density weights are $90 \%-10 \%$

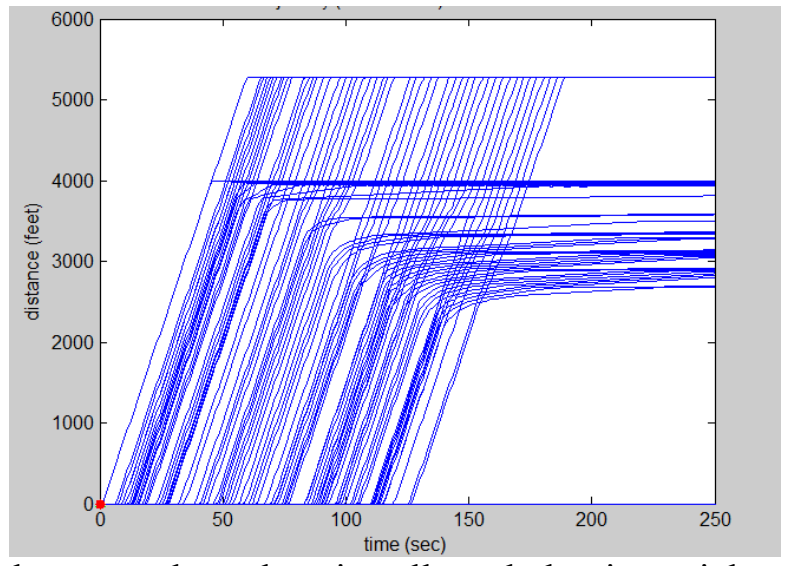

Figure 13: Work zone no lane changing allowed; density weights are $90 \%$ - 10\%

\section{CONCLUSION}

In this paper, the lane-changing enhanced Anisotropic Mesoscopic Simulation (AMS) model is presented and discussed to account for special scenarios involving stalled or particularly slow-moving vehicles. This is the first step toward the concept of Adaptive Simulation (AS) in which the lane-based simulation is switch on depending on the need to depict traffic dynamic in a greater level of details under such circumstance. The underlying simulation rules and macroscopic dynamical characteristics presented in this paper show desirable lane-changing properties. This enables a computationally efficient AMS to deal with situations in which more microscopic details are needed in a dynamic and adaptive manner. This enhancement allows AMS to retain its computational efficiency to support large-scale transportation and logistics modeling with realistic traffic dynamic properties.

\section{REFERENCES}

Alexiadis, V. 2008. Integrated corridor management analysis, modeling, and simulation results for the test corridor. Cambridge Systematics, Inc. 
Ben-Akiva, M., M. Bierlaire, D.r Burton, H. N. Koutsopoulos, and R. Mishalani. 2001. "Network State Estimation and Prediction for Real-Time Traffic Management." Networks and Spatial Economics 1 (2):293-318.

Ben-Akiva, M., D. Cuneo, M. Hasan, M. Jha, and Q. Yang. 2003. "Evaluation of freeway control using a microscopic simulation laboratory." Transportation Research Part C 11:29-50.

Bloomberg, L., and J. Dale. 2000. "Comparison of VISSIM and CORSIM Traffic Simulation Models on a Congested Network." Transportation Research Record 1727:52-60.

Botma, H. 1978. State-of-the-Art " Traffic Flow Models". In Research Report R-78-40: SWOV.

Branston, D. 1976. "Models of Single Lane Time Headway Distributions." Transportation Science 10 (2):125-148.

Buckley, D. J. 1968. “A Semi-Poisson Model of Traffic Flow.” Transportation Science 2 (2):107-132.

Burghout, W., H. Koutsopoulos, and I. Andréasson. 2005. "Hybrid Mesoscopic-Microscopic Traffic Simulation." Transportation Research Record: Journal of the Transportation Research Board 1934 (1):218-255.

Cetin, N., K. Nagel, B. Raney, and A. Voellmy. 2002. "Large-scale Multi-agent Transportation Simulations." Computer Physics Communications 147 (1-2):559-564.

Cheu, R. L., J. Martinez, and C. Duran. 2009. "A Cell Transmission Model with Lane Changing and Vehicle Tracking for Port of Entry Simulations." Transportation Research Record (241-248).

Cheu, R. L., Y. Tan, and D.-H. Lee. 2004. "Comparison of PARAMICS and GETRAM/AIMSUN Microscopic Traffic Simulation Tools." Paper read at 83rd Annual Meeting of the Transportation Research Board, Washington, D.C.

Chiu, Y.-C., L. Zhou, and H. B. Song. 2010. "Development and calibration of the Anisotropic Mesoscopic Simulation model for uniterrupted flow facilities." Transportation Research Part B 44:152-174.

Daganzo, C. F. 1995. "Requiem for Second-Order Fluid Approximations of Traffic Flow." Transportation Research Part B 29 (4):277-286.

Dell'Orco, M. 2006. "A dynamic network loading model for mesosimulation in transportation systems." European Journal of Operational Research 175 (3):1447-1454.

Ferreira, P., E. Esteves, R. Rossetti, and E. Oliveria. 2009. A"pplying situated Agents to Microscopic Traffic Modelling." In Multi-Agent Systems for Traffic and Transportation Engineering, edited by A. Bazzan and F. Klugl.

Gawron, C. 1998. "An iterative algorithm to determine the dynamic user equilibrium in a traffic simulation model." International Journal of Modern Physics C 9 (3):393-407.

Jayakrishnan, R., H. S. Mahmassani, and T. Y. Hu. 1994. "An Evaluation Tool for Advanced Traffic Information and Management System in Urban Networks." Transportation Research 2C (3):129-147.

Kary, B., V. Papayannoulis, D. Deeter, L. Head, M. Hickman, and Y.-C. Chiu. 2009. "Minneapolis Integrated Corridor Management Pioneer Site Concept and Strategy Modeling - Part 1: Modeling Lessons Learned." In TRB Integrated Corridor Management Conference. Irvine, CA: TRB.

Kerner, B. S., S. L. Klenov, A. Hiller, and H. Rehborn. 2006. "Microscopic features of moving traffic jams." Physical Review E 73:1-16.

Kuhn, B, K. Balke, N. Chaudhary, D. Jasek, G. Karkee, K. Obeng-Boampong, J. Shelton, and S. Venglar. 2008. "Managed Lanes Strategies Feasible for Freeway Ramp Applications." College Station, TX: Texas Transportation Institute.

Laval, J. A., and C. F. Daganzo. 2006. "Lane-changing in traffic streams." Transportation Research Part $B$ 40:251-264.

Mahmassani, H. S., S. Peeta, T.-Y. Hu, and A. K. Ziliaskopoulos. 1993. "Dynamic Traffic Assignment with Multiple User Classes for Real-Time ATIS/ATMS Applications." Paper read at ATMS Conference on Management of Large Urban Traffic Systems, St. Petersburg.

Mahmassani, H. S. 2001. "Dynamic Network Traffic Assignment and Simulation Methodology for Advanced System Management Applications" Networks and Spatial Economics 1 (3-4):267 - 292.

McGraw-Hill, and S. P. Parker. 2002. Dictionary of Scientific and Technical Terms: McGraw-Hill. 
Nagel, K. 1996. "Particle hopping models and traffic flow theory." Physical Review E (53):4655-4670.

Nagel, K, P. Stretz, M. Pieck, S. Leckey, R. Donnelly, and C. L. Barrett. 1997. "TRANSIMS traffic flow characteristics." TRB paper (981332).

Nelson, P., and A. Sopasakis. 1998. "The Prigogine-Herman Kinetic Model Predicts Widely Scattered Traffic Flow Data at High Concentrations." Transportation Research Part B 32 (8):589-604.

Noh, H., Y.-C. Chiu, H. Zheng, M. Hickman, and P. Mirchandani. 2009. "An Approach to Modeling Demand and Supply for a Short-Notice Evacuation." Transportation Research Record Forthcoming.

Ozbay, K., B. O. Bartin, and S. Mudigonda. 2006. "Microscopic Simulation and Calibration of an Integrated Freeway and Toll Plaza Model." In 85th Annual Meeting of the Transportation Research Board. Washington, D. C.

Park, Byungkyu, Jongsun Won, and Ilsoo Yun. 2006. "Application of Microscopic Simulation Model Calibration and Validation Procedure: A Case Study of Coordinated Actuated Signal System." Transportation Research Record 1978:113-122.

Shelton, J. 2007. IH-10 East Corridor Improvement Study using Multi-Resolution Dynamic Traffic Simulation Approach - Final Report. El Paso: Texas Transportation Institute.

Shelton, J. 2008. "Getting More by Working Together - Integrating Mesoscopic DTA and Microscopic Models." In PTV User Group Meeting: PTV America.

Shelton, Jeffrey, and Yi-Chang Chiu. 2009. "Toward a Consistent and Robust Integrated MultiResolution Modeling Approach for Traffic Analysis." Paper read at 2009 TRB Planning Application Conference, Houston, Texas.

Simith, M. C., and A. W. Sadek. 2006. Challenges Calibrating a Large-Scale Microscopic Simulation Model of a Diverse Urban, Suburban, and Rural Netowrk: A Practical Guide. In 85th Annual Meeting of the Transportation Research Board. Washington, D. C.

Taylor, N.B. 2003. "The CONTRAM Dynamic Traffic Assignment Model." Networks and Spatial Economics 3 (3):297-322.

Yang, Q., and H. N. Koutsopoulos. 1996. "A Microscopic Traffic Simulator for evaluation of dynamic traffic management systems." Transportation Research Part C 4 (3):113-129.

Yin, Y. H. X. Liu, J. A. Laval, and X.-Y. Lu. 2007. "Development of an Integrated Microscopic Traffic Simulation and Signal Timing Optimization Tool." Berkeley, CA: California PATH at the University of California at Berkeley.

Zhang, H. M. 2001. "New Perspectives on Continuum Traffic Flow Models." Networks and Spatial Economics 1 (1-2):9-33.

Zhang, Y., P. Yi, K. Kreider, C. Shao, and T. Norfolk. 2010. "Modeling Network Impact in Area Surrounding Activity Center Caused by Special Events." Transportation Research Record: Journal of the Transportation Research Board 2183 (-1):78-84.

Zheng, H., Y.-C. Chiu, P. Mirchandani, and M. Hickman. 2010. "Modeling of Evacuation and Background Traffic for an Optimal Zone-Based Vehicle Evacuation Strategy." Transportation Research Record forthcoming.

\section{AUTHOR BIOGRAPHIES}

YE TIAN is a doctoral student in the Department of Civil Engineering And Engineering Mechanics at the University of Arizona, majoring in Transportation Engineering under Dr. Yi-Chang Chiu. He received a Bachelor degree of Engineering from Zhejiang University, Hangzhou, China in 2010. He has published several papers about Parking Guide Information Systems. He is a student member of ITE. His research interests are in traffic simulation, shortest path algorithms and other network modeling problems. His email address is tianye@email.arizona.edu.

YI-CHANG CHIU is an Associate Professor in the Department of Civil Engineering and Engineering Mechanics at the University of Arizona (UA). He received his Ph.D. degree in transportation engineering from the University of Texas at Austin in 2002. Prior to joining UA in 2006, he served as an Assistant 
Professor at the University of Texas at El Paso (UTEP), research engineer at the Center for Transportation Research at UT-Austin, and a senior technical staff member at Nortel Networks Inc. in Richardson, Texas. Dr. Chiu has developed open-source DTA model system called DynusT (Dynamic Urban Systems in Transportation) has been used by more than 50 agency users internationally. Prof. Chiu is the member of the TRB ADB30 Transportation Network Modeling Committee and editor board member of ASCE Journal of Urban Planning and Development and International Journal of Transportation Science and Technology. His email address is chiu@email.arizona.edu. 